\title{
Analytical study of the edge states in the bosonic Haldane model
}

\author{
Pierre A. Pantaleón and Y. Xian \\ Theoretical Physics Division, School of Physics and Astronomy, \\ University of Manchester, Manchester M13 9PL, United Kingdom
}

\begin{abstract}
We investigate the properties of magnon edge states in a ferromagnetic honeycomb spin lattice with a Dzialozinskii-Moriya interaction (DMI). We derive analytical expressions for the energy spectra and wavefunctions of the edge states localized on the boundaries. By introducing an external on-site potential at the outermost sites, we show that the bosonic band structure is similar to that of the fermionic graphene. We investigate the region in the momentum space where the bosonic edge states are well defined and we analyze the width of the edge state and their dependence with the DMI strength. Our findings extend the predictions using topological arguments and they allow size-dependent confirmation from possible experiments.
\end{abstract}

\section{INTRODUCTION}

Perhaps the most important and intriguing aspect of the topological insulators is the presence of edge states. A well known example is the Kane-Mele model in graphene [1], where the spin-orbit coupling (SOC) causes a transition from a semi-metal to a quantum spin Hall insulator. Accompanying the transition is the appearing of gapless edge states with distinct properties from the bulk energy band [2-4]. In a fermionic model, the edge states are spin-filtered, since the electrons with opposite spins propagate in opposite directions with robustness against external perturbations $[5,6]$.

The edge states have also been studied in magnetic insulators [7], where the spin moments are carried by magnons. The magnon Hall effect was observed in a collinear ferromagnetic insulator $\mathrm{Lu}_{2} \mathrm{~V}_{2} \mathrm{O}_{7}$ with pyrochlore structure [8], in the Kágome ferromagnetic lattice [9], and have also been studied in the Lieb lattice [10] and honeycomb ferromagnetic lattice [11].

Until recently, most investigations of the edge states have been based on fermionic models. Now similar study has been extended to the bosonic models. For example, it has been recently shown that the bosonic equivalent for the Kane-Mele-Haldane model is a ferromagnetic Heisenberg Hamiltonian with the Dzialozinskii-Moriya interaction. The thermal Hall effect [12] and spin Nernst effect [13] have been predicted for this magnetic system. So far, the study of the magnon edge states is mainly based on topological arguments and clearly the detailed properties of the edge states will be required if the potential of magnonics is to be realized [14]. In particular, systematic investigation of the spin-density profile (or magnon density) of the edge magnon and their dependence with the DMI strength and external on-site potentials may be useful in manufacturing small-sized devices based on such magnets.

Although a direct experimental demonstration of the magnon edge modes in magnetic systems is still absent, it is interesting to ask whether the similar edge properties in the fermionic systems are also exhibited in the bosonic counterparts. Particularly, with regard to the fact that the strength of DMI in some magnets is greater than that of SOC in graphene by five orders of magnitude $[8,9]$. With such motivations in this paper we analyze a ferromagnetic honeycomb lattice with DMI. After introducing an on-site potential at the outermost sites [15, 16] we derive an analytical form for the wavefunctions and energy spectrum for the edge states. We investigate the conditions in the momentum space where the edge states are well defined and we write the edge-state width in terms of the DMI strength.

This paper is organized as follows: In Sec. II we introduce the model Hamiltonian with a next-nearest neighbor DMI which opens a gap in the energy spectra. In Sec. III we adapt the analytical approach for the edge state developed by Wang. et. al. [17] and Doh. et. al [18, 19] to a bosonic Haldane model. In order to cover the region where most study has been focusing using topological approach, we introduced an on-site potential at the outermost sites. In Sec. IV, the edge states and their energy spectra for zero and non-zero DMI are investigated. We also analyze the range in the momentum space in which the edge states are well defined and we express the width of the edge state in terms of the DMI strength. We conclude with a summary in Sec. V. A brief description to obtain the edge states and the energy spectrum without DMI is presented in the appendix.

\section{MODEL HAMILTONIAN}

We consider a ferromagnetic Heisenberg Hamiltonian with DMI on a honeycomb lattice. The DMI vanishes for the nearest-neighbors (NN) but has non-zero component along the $z$-direction for the next-nearest-neighbors (NNN) [20]. Assuming $\vec{D}=D \hat{\mathbf{z}}$, the corresponding model Hamiltonian for $J>0$ is

$$
H=-J \sum_{\langle i, j\rangle} \mathbf{S}_{i} \cdot \mathbf{S}_{j}+D \sum_{\langle\langle i, j\rangle\rangle} \nu_{i j} \hat{\mathbf{z}} \cdot\left(\mathbf{S}_{i} \times \mathbf{S}_{j}\right),
$$


where $\langle i, j\rangle$ indicates the $\mathrm{NN}$ coupling, $\langle\langle i, j\rangle\rangle$ is the NNN coupling, in analogy with the Kane-Mele model, and $\nu_{i j}= \pm 1$, depending on the orientation of the two sites [1]. The energy spectrum of Eq. (1) is given by

$$
\varepsilon=3 J S\left(1 \pm \sqrt{h_{d}^{2}+\left|\gamma_{k}\right|^{2}}\right)
$$

in the linear spin-wave approximation for the infinite system. Here, $\gamma_{k}=\frac{1}{3} \sum_{\sigma} \exp (k \cdot \sigma)$ is the honeycomb complex structure factor and $h_{d}=\frac{2}{3} \frac{D}{J} \sum_{\mu} \sin (k \cdot \mu)$ is the contribution due to the DMI. The index vectors $\sigma$ and $\mu$ run over the three NN and NNN with positive hopping term, as shown in Fig. (1). The lower energy band becomes negative if $\frac{D}{J}>\frac{1}{\sqrt{3}}$, which is a signature of a phase transition in the system [13]. Here, we only consider the case when $D<\frac{J}{\sqrt{3}}$ with the ferromagnetic ground state.

The system of Eq. (1) has a non-trivial band topology. The DMI induces a non-trivial gap of $\frac{6 \sqrt{3} D}{J}$ at the two Dirac points $\mathbf{K}$ and $\mathbf{K}^{\prime}$ in the spin-wave spectra and edge states emerge with gapless energy [11]. Motivated by such predictions, in the following sections, we derive analytical expressions for the edge states and explicit forms for the energy spectrum for the semi-infinite system.

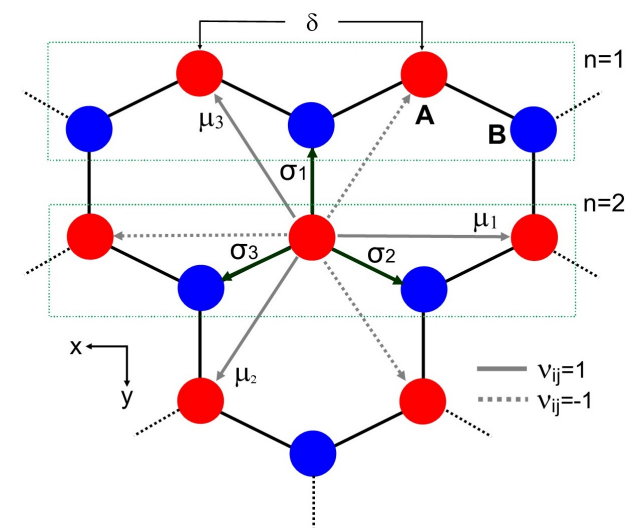

Figure 1. (Color on-line) Schematic of a honeycomb lattice with zig-zag edges. $\sigma, \mu$ are the nearest-neighbors and the next-nearest neighbor index vectors, respectively. $\nu_{i j}= \pm 1$ is the orientation dependent coefficient and is positive (negative) if the electron makes a left (right) turn to get the second site. The external on-site potential $\delta=1$ is introduced at the outermost sites.

\section{EDGE STATES AND BOUNDARY CONDITIONS}

\section{A. Hamiltonian matrix elements}

Starting from a Neél ordering state for a bipartite lattice and by the standard Holstein-Primakoff transformations in the linear spin-wave theory, we can write the following effective bosonic Hamiltonian for a ferromagnetic lattice of Eq. (1) as,

$$
\begin{aligned}
H= & -J S \sum_{i, \sigma}\left(a_{i} b_{i+\sigma}^{\dagger}+a_{i}^{\dagger} b_{i+\sigma}-a_{i}^{\dagger} a_{i}-b_{i+\sigma}^{\dagger} b_{i+\sigma}\right) \\
& +i D S \sum_{i, \mu} \nu_{i, i+\mu}\left(a_{i} a_{i+\mu}^{\dagger}-a_{i}^{\dagger} a_{i+\mu}\right) \\
& +i D S \sum_{j, \mu} \nu_{j, j+\mu}\left(b_{j} b_{j+\mu}^{\dagger}-b_{j}^{\dagger} b_{j+\mu}\right)
\end{aligned}
$$

where the index $i(j)$ denotes the $A(B)$ sublattice sites, $\sigma$ and $\mu$ are the NN and NNN vector, respectively, as shown in Fig. (1). This Hamiltonian is the bosonic equivalent to the Haldane model [21]. To investigate the edge state, we assume a zigzag boundary along the $x$ direction and semi-infinite in the $y$ direction. If $k$ is the momentum in the $x$ direction, the Hamiltonian (3) can be written as,

$$
H=J S \sum_{k} \Psi^{\dagger} M \Psi
$$

where $\Psi_{k}^{\dagger}=\left[\Psi_{k, A}^{\dagger}, \Psi_{k, B}^{\dagger}\right]$ is a semi-infinite 2-component spinor. The matrix elements of $M$ are semi-infinite matrices: $M_{11}=(2+\delta)+(1-\delta) T^{\dagger} T+J_{3}-J_{4}\left(T+T^{\dagger}\right)$, $M_{12}=-\left(J_{1}+J_{2} T^{\dagger}\right), M_{21}=M_{12}^{\dagger}$ and $M_{22}=(2+\delta)+$ $(1-\delta) T T^{\dagger}-J_{3}+J_{4}\left(T+T^{\dagger}\right)$, where $J_{1}=2 \cos \left(\frac{\sqrt{3}}{2} k\right)$, $J_{2}=1, J_{3}=2 D^{\prime} \sin (\sqrt{3} k), J_{4}=2 D^{\prime} \sin \left(\frac{\sqrt{3}}{2} k\right)$, $D^{\prime}=D / J$, and $T$ is a semi-infinite displacement matrix as defined in Ref. [22]. Here, we introduced an external on-site potential, $\delta$, at the outermost sites. In a ferromagnetic lattice the intrinsic on-site potential is given by the number of nearest-neighbors, along the zig-zag edge there is a missing bond and the edge on-site energy is lower than in the bulk. By introducing an external onsite potential the edge state can be modified [5, 15, 23]. In this work, we will focus on the "symmetric" case with $\delta=1$, as in Refs. [11-13], where the on-site potential along the edge is the same as in the bulk. We will consider other cases with different values of $\delta$ elsewhere.

\section{B. Self-consistent equation for the edge modes}

From the Hamiltonian (4) and with the explicit form of the matrix elements, we can construct the coupled Harper equations [24, 25] for the edge state. Using the assumption that the edge states are exponentially decaying from the boundary, we consider the following anzats $[17,26]$ for the eigenstates of $M$ in Eq. (4),

$$
\psi_{k}(n)=z^{n} \phi_{k}
$$

In the above wavefunction $\phi_{k}$ is a 2 -component vector, $z$ is a complex number with magnitude less than the unity and $n=1,2,3 \ldots$, are the lattice coordinates in the $y$ direction as shown in Fig. (1). The form $z^{n}$ in the above 
equation is a finite-size Fourier transform along the $y$ direction and is related to the eigenvalues of a finite-size displacement matrix [22]. Therefore, the effective Hamiltonian for the edge state can now be written as,

$$
H=\left[\begin{array}{cc}
3+J_{3}-J_{4}\left(z+z^{-1}\right) & -\left(J_{1}+J_{2} z^{-1}\right) \\
-\left(J_{1}+J_{2} z\right) & 3-J_{3}+J_{4}\left(z+z^{-1}\right)
\end{array}\right] .
$$

The characteristic equation for the eigenvalue $\varepsilon$ is obtained from the condition, $|H(z)-\varepsilon|=0$, or,

$$
a\left(z+z^{-1}\right)^{2}+b\left(z+z^{-1}\right)+c=0
$$

where, $a=-J_{4}^{2}, b=2 J_{3} J_{4}-J_{1} J_{2}, c=(\varepsilon-3)^{2}-J_{1}^{2}-$ $J_{2}^{2}-J_{3}^{2}$. We solve for $z+z^{-1}$ to obtain four solutions $z_{1}^{ \pm}$and $z_{2}^{ \pm}$in terms of the momentum $k$ and energy $\varepsilon$. Two of the solutions with $|z|<1$ describe the state at the upper edge, while the other two with $|z|>1$ describe the state for the lower edge, if any. The eigenfunction of Eq. (4) satisfying $\lim _{n \rightarrow \infty} \psi_{k}(n)=0$ may now in general be written as,

$$
\psi_{k}(n)=u_{1} z_{1}^{n} \phi_{1}+u_{2} z_{2}^{n} \phi_{2}
$$

where $u_{1(2)}$ are normalization constants and the eigenvector of the Hamiltonian, Eq. (6), corresponding to the solution $z_{r}(r=1,2)$, is given by

$$
\phi_{r}=\left[\begin{array}{c}
J_{1}+J_{2} z_{r}^{-1} \\
3-\varepsilon+J_{3}-J_{4}\left(z_{r}+z_{r}^{-1}\right)
\end{array}\right] .
$$

If an opposite boundary exists, the semi-infinite matrices in Eq. (4) can be truncated at some size $N$ and the edge states of the system will become a linear combination of the solutions at each boundary [3]. As $N$ increases over some value, the edge states become independent of the size as expected [27, 28], allowing us to obtain the solutions at each edge independently. For a large $N$ and for the opposite boundary, the edge state can be obtained if we perform the substitution $z^{n} \rightarrow z^{N-n+1}$ in the Eq. (8). Furthermore, we have to choose the eigenvector whose $A$-sublattice component goes to zero when $D \rightarrow 0$ (the details of the solution without DMI are presented in the appendix). From the Hamiltonian (6), the eigenvector, $\phi_{r}^{\prime}$, for the opposite edge, has the form,

$$
\phi_{r}^{\prime}=\left[\begin{array}{c}
3-\varepsilon-J_{3}+J_{4}\left(z_{r}+z_{r}^{-1}\right) \\
J_{1}+J_{2} z_{r}
\end{array}\right] .
$$

For a zig-zag edge, the boundary conditions are satisfied by setting:

$$
\psi_{k}^{\prime}(N+1)=\psi_{k}(0)=0
$$

As in the Kane-Mele model, the eigenvectors for each solution are linearly dependent [19]. Then, from the Eq. (8) and the edge condition, Eq. (11), the non-trivial solution is given by setting the determinant $\left|\phi_{1} \phi_{2}\right|=0$ or $\left|\phi_{1}^{\prime} \phi_{2}^{\prime}\right|=0$ for the other edge, providing us the following additional relation for the edge energy dispersion,

$$
\varepsilon-3-2 J_{3}+J_{4}\left(z_{1}+z_{2}\right)+J_{3} z_{1} z_{2}=0,
$$

with eigenstates

$$
\psi_{k}(n)=u_{1}\left(z_{1}^{n}-z_{2}^{n}\right) \phi_{1}
$$

for the upper edge. As we mentioned before, for the lower edge at large $N$, the eigenstate and energy spectrum can be obtained by the substitutions $z^{n} \rightarrow z^{N-n+1}$ in the Eq. (13) and $z \rightarrow z^{-1}$ in the Eq. (12).

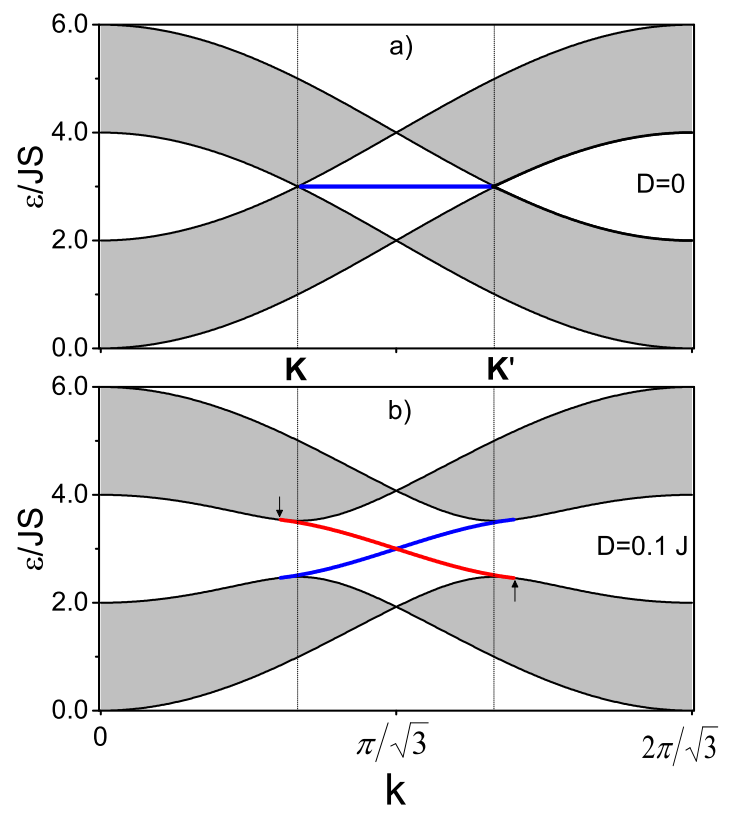

Figure 2. (Color online) Energy spectrum for a honeycomb ferromagnetic lattice with DMI and external on-site potential $\delta=1$ at the outermost sites. a) For $D=0$, the horizontal line at $\varepsilon=3 J S$ is the flat spectrum for the edge state. b) For $D=0.1 J$, the two bands crossing the gap near the Dirac points are the chiral edge states for the upper (red) and lower (blue) edges. The arrows show the critical points at $k_{l}$ before $\mathbf{K}$ and $k_{r}$ after $\mathbf{K}^{\prime}$ (see text).

\section{RESULTS AND DISCUSSIONS}

\section{A. Energy Spectrum}

Equations (7) and (12) can be solved numerically for the energy and the wavefunction of the edge state. We first discuss the case of $D=0$. As shown in Fig. 2(a), the bulk energy spectra is always degenerate at the $\mathbf{K}$ and $\mathbf{K}^{\prime}$ points, independently of the boundary conditions. However, if we consider a semi-infinite lattice with zig-zag edges, a flat band appears (see appendix for further details). Such flat band is the bosonic analogous to the edge 
state in zig-zag graphene $[29,30]$ and the corresponding edge state can be described in a similar way as follows: the bulk bands for a ferromagnetic lattice are symmetric around $\varepsilon=3$, hence, if a state exists along the boundary, the sum of the components of the wavefunction over the nearest-neighbors sites must be equal to the on-site energy. Such equality is only fulfilled if we include an external on-site energy, in particular for $\delta=1$ we obtain an edge state localized at the $A$-sublattice. From the Eq. (A5), we can write the wavefunction as,

$$
\psi_{k}(n) \propto\left(-J_{1}\right)^{n-1},
$$

where $J_{1}$ is a function of $k$ as we defined before. Hence, the spin-density of the edge magnon is proportional to $J_{1}^{2(n-1)}$ in the region $\frac{2 \pi}{3 \sqrt{3}} \leq k \leq \frac{4 \pi}{3 \sqrt{3}}$ (between the $\mathbf{K}$ and $\mathbf{K}^{\prime}$ points) where the flat band exists. At the point $k_{0}=\frac{\pi}{\sqrt{3}}$ the edge state is completely localized at the single line of boundary sites and gradually extends inside to the inner sites as we move to the Dirac Points where it merges into the bulk. For the opposite edge, with the same argument, an edge state exists and is localized at the $B$-sublattice.

We next consider the case of non-zero DMI. The energy bands are obtained by solving the Eq. (12) with the solutions given by the Eq. (7), and the edge wavefunction by the Eq. (13). In the Fig. 2(b) we show the energy bands for a DMI strength of $D=0.1 J$. The shaded regions correspond to the bulk spectra, while the two bands which transverse the gap, connecting the upper and lower bulk bands, are the spectra of the edge states. By the analysis of the Eq. (12) or the wavefunctions of Eq. (13), we find that there is only one edge state at each boundary. The edge band at the upper boundary has negative slope while the edge band at the opposite edge has positive slope. Hence, as predicted in Ref. [11], the magnons are moving to the left at one of the edges, while they are moving to the right in the other one. On the other hand, at the point $k_{0}=\frac{\pi}{\sqrt{3}}$ and $\varepsilon=3$, the edge state for the upper edge takes a simple form,

$$
\psi_{k_{0}}(n) \propto\left[1-(-1)^{n}\right]\left[\begin{array}{c}
z_{0}^{-1} \\
i
\end{array}\right] z_{0}^{n},
$$

where $z_{0}=\frac{i}{4 D^{\prime}}\left(-1+\sqrt{1+16 D^{\prime 2}}\right)$. The spin-density of the edge magnon is therefore proportional to $\left|z_{0}\right|^{2(n-1)}$ and $\left|z_{0}\right|^{2 n}$ for the $A$ and $B$ sublattice, respectively, decaying exponentially. As we move from $k_{0}$ to near the Dirac points $\mathbf{K}$ or $\mathbf{K}^{\prime}$, the edge state becomes delocalized and merged with that of the bulk state.

\section{B. Allowed range for the edge states}

We notice that the restriction $\left|z_{1,2}\right|<1$ in the Eq. (12) and (13) allow us to investigate two important properties of the edge states: the region in the momentum space where they are well defined and their confinement to the physical boundary. In general, a particular edge state has two critical points defined as $k_{l}$ and $k_{r}$ located before and after the two Dirac $\mathbf{K}$ and $\mathbf{K}^{\prime}$ points, respectively, as indicated in the Fig. 2(b). For the upper edge, the eigenfunctions (and also the energy spectrum) depends on $z_{1}^{+}$and $z_{2}^{+}$in the (left) interval $k \in\left(k_{l}, k_{0}\right)$ and on $z_{1}^{-}$ and $z_{2}^{-}$in the (right) interval $k \in\left(k_{0}, k_{r}\right)$. The critical points are determined when the modulus of one $z$ in Eq. (12) reach the unity and the edge state becomes indistinguishable from the bulk. In the Fig. (3) the plot of $|z|$ versus $k$ is shown for different values of $D$ in the interval where the edge state is well defined. For the edge state at the upper edge, the critical point near $\mathbf{K}$ is given when the modulus $\left|z_{2}^{+}\right|$, reach the unity, whereas that in the right interval (near $\mathbf{K}^{\prime}$ ), the critical point is given when $\left|z_{1}^{-}\right|=1$. In particular for $D=0.1 J, k_{l} \approx 1.09$ and $k_{r} \approx 2.53$ [See. Fig. 2(b)]. Only when $D=0$, these critical points correspond to the Dirac points.

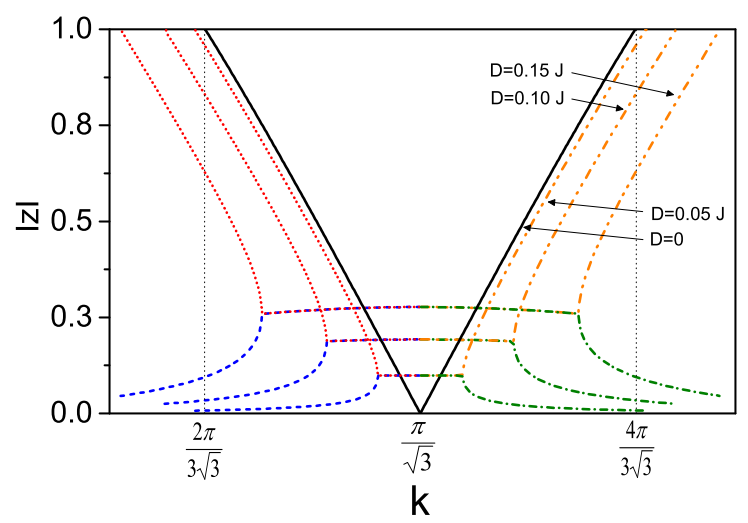

Figure 3. (Color on-line) $|z|$ vs $k$ at different values of $D$. The solutions correspond to $\left|z_{1}^{+}\right|$(dashed, blue), $\left|z_{2}^{+}\right|$(dot, red), $\left|z_{2}^{-}\right|$ (dot-dashed, green) and $\left|z_{1}^{-}\right|$(dot-dot-dashed, orange). The continuous line (black) corresponds to $z=-J_{1}$ for $D=0$.

If we increase the value of $D$, the critical points move further away from the Dirac points as shown in Fig. (3). This suggests that the edge state merges into the bulk but remains in (or near) its boundaries. Such critical points are important since they define the range where the edge state is well defined and they also determine the critical region where $z$ is transformed from real to a complex with unitary modulus. The $z$ solutions with unitary modulus, which obey the bulk and boundary conditions, are sensitive to the values of DMI and the details of edge modifications.

\section{Width of the bosonic edge state}

In the previous section, we have investigated the critical points and their dependence with the solutions $z_{2}^{+}$and 
$z_{1}^{-}$in their corresponding intervals. In this section, we will examine the width of the edge state and their relation with the remaining solutions, $z_{1}^{+}$and $z_{2}^{-}$. As described before, the spin-density of the edge magnon decreases as we move away from the boundary, and this happens with some characteristic length scale. Following the Ref. [18] for a semi-infinite zig-zag graphene, the width or characteristic length scale is given by,

$$
\xi_{i}(k) \equiv \frac{3}{2}\left[\ln \left|\frac{1}{z_{i}(k)}\right|\right]^{-1},
$$

where $\left|z_{i}(k)\right|<1$ is the smallest factor in the eigenstate given by Eq. (13). We notice that such condition is fulfilled by $z_{1}^{+}$and $z_{2}^{-}$between $\left(k_{l}, k_{0}\right)$ and $\left(k_{0}, k_{r}\right)$, respectively, as shown in Fig. (3). In Fig. (4) we plot the width versus momentum for different values of $D$, where by completeness we also have included the larger decaying factors. In the slightly flat region around $k_{0}$ the width is almost constant. Here, $\left|z_{1}^{+}\right|=\left|z_{2}^{+}\right|$and $\left|z_{1}^{-}\right|=\left|z_{2}^{-}\right|$, since the discriminant of the Eq. (7) is negative $\left(b^{2}-4 a c<0\right)$, which gives rise a two pairs of complex conjugate solutions, one pair with magnitude lower than one. However, out of this complex region and between the critical values, the solutions are real. In such region, the width associate to the solution $z_{1}^{+}$decreases while the associate to $z_{2}^{+}$grows quickly and diverges at the critical point where the edge state merges into the bulk. Since the edge state wave-function is written as a linear combination, Eq. (13), then the edge state merges into the bulk through a bifurcation of the edge state width in complete analogy with a semi-infinite graphene $[18,19]$.

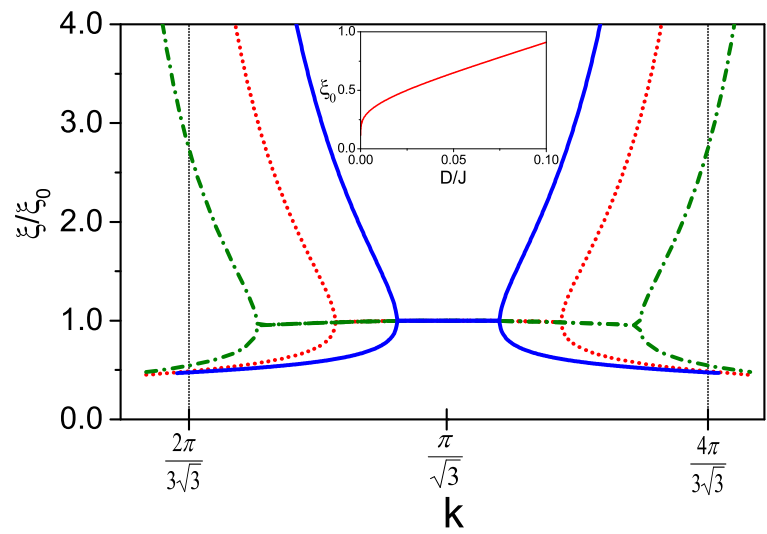

Figure 4. (Color on-line) Edge state width $\xi$ vs $k$ at different values of $D$. The DMI values correspond to: $D=0.05 J$ (continuous, blue), $D=0.10 \mathrm{~J}$ (dotted, red) and $D=0.15 \mathrm{~J}$ (dash-dotted, green). Inset: Edge state width at $k_{0}=\frac{\pi}{\sqrt{3}}$ as a function of $D / J$.

The inset in Fig. (4) shows the edge state width as a function of $D$ at the point $k_{0}$. At such point the width can be obtained explicitly. From the Eq. (12) for $k=k_{0}$ we obtain $z_{1}^{+}=-z_{2}^{-}$for $\varepsilon=3$, and by the Eq. (16) we can write

$$
\xi_{0}=\frac{3}{2}\left\{\ln \left[\frac{4(D / J)}{\sqrt{1+16\left(\frac{D}{J}\right)^{2}}-1}\right]\right\}^{-1} .
$$

Expanding near $D=0$ the above equation takes the form,

$$
\xi_{0} \approx-\frac{3}{2}\left[\ln \left[\frac{2 D}{J}\right]\right]^{-1},
$$

which shows that the width of the edge state vanishes logarithmically as the DMI approaches to zero. Such behavior can be also observed in the Fig. (3), where the $z$ solutions collapses to a single, $z=-J_{1}$. In consequence, at $D=0$ and $k=k_{0}$ the edge state is completely localized at the single line of boundary sites as we mentioned before.

As in the fermionic model, the width of the edge state as we discussed above could have important effects when determining the edge properties of samples with finite size [31-33]. For example, it has been found that there is magnon propagation in interfaces between ferromagnets and normal metals with a strong spin-orbit coupling, referred to as interfacial DMI interaction [34-36]. It also have been proposed magnon waveguides, where the propagation is between the interface of two topological insulators [37]. In both such cases, the width of the edge state will be useful in the measurement of the magnon current.

\section{CONCLUSIONS}

We have derived analytical expressions for the magnon edge-state wavefunctions and their energy spectra for a honeycomb lattice with DMI and zig-zag edges. We demonstrate that the bosonic edge states are defined in a region in the momentum space before merging into the bulk and the width of the edge state can be controlled by the DMI strength. By introducing an on-site potential at the outermost sites we found that the magnonic ferromagnetic band structure closely resembles that of the fermionic graphene. However, unlike graphene where the energy spectrum is doubly degenerate, our explicit solutions demonstrate that there is only one chiral magnon edge state at each edge as predicted by topological approaches [11-13]. Moreover, our approach is applicable for arbitrary values of the on-site potential $\delta$ as introduced in Eq. (4). The edge-magnon width, the chirality and the spin density as a function of the DMI presented in this paper could be useful for experiments in small sized monolayers or thin film magnets.

\section{ACKNOWLEDGMENTS}

We thank Wissam A. Ameen, R. Carrillo-Bastos and R. Romo for discussions. Pierre. A. Pantaleón is sponsored 
by Mexico's National Council of Science and Technology (CONACYT) under the scholarship No. 381939.

\section{Appendix A: Analytical Solutions for the Hamiltonian with $\mathrm{D}=0$}

In this appendix, we derive the edge states for $D=0$ with an on-site potential $\delta=1$ at the outermost sites. The coupled Harper equations of the Hamiltonian (4) are given by

$$
\begin{aligned}
3 \psi_{A}(n)-J_{1} \psi_{B}(n)-J_{2} \psi_{B}(n-1) & =\varepsilon \psi_{A}(n), \\
-J_{1} \psi_{A}(n)-J_{2} \psi_{A}(n+1)+3 \psi_{B}(n) & =\varepsilon \psi_{B}(n),
\end{aligned}
$$

where $\psi_{A, B}(n)$ are the eigenfunctions for each sublattice. Using the Eq. (5) we can write the Eq. (A1) in terms of the 2-component vector $\phi$ as,

$$
\left[\begin{array}{cc}
3 & -J_{1}-J_{2} z^{-1} \\
-J_{1}-J_{2} z & 3
\end{array}\right]\left[\begin{array}{l}
\phi_{A} \\
\phi_{B}
\end{array}\right]=\varepsilon\left[\begin{array}{l}
\phi_{A} \\
\phi_{B}
\end{array}\right],
$$

for $n>1$ (and $n<N$, for a large $N$ ). The non-trivial solution gives rise to the characteristic equation,

$$
(3-\varepsilon)^{2}-J_{1}^{2}-J_{2}^{2}-J_{1} J_{2}\left(z+z^{-1}\right)=0,
$$

which is a quadratic equation in $z$. The edge state can be constructed with the solution $|z|<1$ in the Eq. (A3). As in the case for non-zero DMI, we require an additional condition for $z$ and $\varepsilon$. From the Harper equations for the case of $n=1$, such condition is given by,

$$
(3-\varepsilon)^{2}-J_{1}^{2}-J_{1} J_{2} z=0 .
$$

Hence, the existence of an edge state requires that $z$ and $\varepsilon$ must satisfied both equations (A3) and (A4). One such solution is $\varepsilon=3$ and $z=-J_{1}$, with eigenstate

$$
\psi_{k}(n)=z^{n}\left[\begin{array}{c}
\phi_{A} \\
0
\end{array}\right]
$$

which is localized on the $A$-sublattice. This solution is consistent with the Eq. (12) and the eigenstate given by the Eq. (13) in the limit $D \rightarrow 0$.

For a large $N$, we obtain the solution for the opposite edge, by setting $n=N$ in the Harper equations and write

$$
(3-\varepsilon)^{2}-J_{1}^{2}-J_{1} J_{2} z^{-1}=0 .
$$

Together with the bulk equation (A3) we find the solutions as $\varepsilon=3$ for $z=-1 / J_{1}$. Hence, the eigenstate in terms of the ribbon size and the solution for the upper boundary, can be written as

$$
\psi_{k}^{\prime}(n)=z^{N-n+1}\left[\begin{array}{c}
0 \\
\phi_{B}^{\prime}
\end{array}\right] .
$$

Such solution is consistent (in the limit $D \rightarrow 0$ ) with the eigenvector given in Eq. (10). Finally, for the range in the momentum, since we require $|z|<1$ for the upper and $|z|>1$ for the lower edge, the flat edge state exists between, $k \in\left(\frac{2 \pi}{3 \sqrt{3}}, \frac{4 \pi}{3 \sqrt{3}}\right)$ which are the coordinates of the $K$ and $K^{\prime}$ points, [See Fig. 2(a)].
[1] C. L. Kane and E. J. Mele, Phys. Rev. Lett. 95, 226801 (2005).

[2] V. P. Gusynin, V. A. Miransky, S. G. Sharapov, and I. A. Shovkovy, Low Temp. Phys. 34, 778 (2008).

[3] K. Wakabayashi, K.-i. Sasaki, T. Nakanishi, and T. Enoki, Sci. Technol. Adv. Mater. 11, 054504 (2010).

[4] S. Konschuh, M. Gmitra, and J. Fabian, Phys. Rev. B 82, 245412 (2010).

[5] W. Yao, S. A. Yang, and Q. Niu, Phys. Rev. Lett. 102, 096801 (2009).

[6] M. Konig, M. Baenninger, A. G. F. Garcia, N. Harjee, B. L. Pruitt, C. Ames, P. Leubner, C. Brüne, H. Buhmann, L. W. Molenkamp, and D. Goldhaber-Gordon, Phys. Rev. X 3, 021003 (2013).

[7] S. Fujimoto, Phys. Rev. Lett. 103, 047203 (2009).

[8] Y. Onose, T. Ideue, H. Katsura, Y. Shiomi, N. Nagaosa, and Y. Tokura, Science 329, 297 (2010).

[9] R. Chisnell, J. S. Helton, D. E. Freedman, D. K. Singh, R. I. Bewley, D. G. Nocera, and Y. S. Lee, Phys. Rev. Lett. 115, 147201 (2015).

[10] X. Cao, K. Chen, and D. He, J. Phys. Condens. Matter 27, 166003 (2015).
[11] S. A. Owerre, J. Phys. Condens. Matter 28, 386001 (2016).

[12] S. A. Owerre, J. Appl. Phys. 120, 043903 (2016).

[13] S. K. Kim, H. Ochoa, R. Zarzuela, and Y. Tserkovnyak, Phys. Rev. Lett. 117, 227201 (2016).

[14] V. V. Kruglyak, S. O. Demokritov, and D. Grundler, J. Phys. D. Appl. Phys. 43, 264001 (2010).

[15] R. Sakaguchi and M. Matsumoto, J. Phys. Soc. Japan 85, 104707 (2016).

[16] H. Guo, Y. Niu, S. Chen, and S. Feng, Phys. Rev. B 93, 121401 (2016).

[17] Z. Wang, N. Hao, and P. Zhang, Phys. Rev. B 80, 115420 (2009).

[18] H. Doh and G. S. Jeon, Phys. Rev. B 88, 245115 (2013).

[19] H. Doh, G. S. Jeon, and H. J. Choi, arXiv:1408.4507v1 (2014).

[20] T. Moriya, Phys. Rev. 120, 91 (1960).

[21] F. D. M. Haldane, Phys. Rev. Lett. 61, 2015 (1988).

[22] J.-S. S. You, W.-M. M. Huang, and H.-H. H. Lin, Phys. Rev. B 78, 161404 (2008).

[23] G. W. Semenoff, V. Semenoff, and F. Zhou, Phys. Rev. Lett. 101, 087204 (2008). 
[24] P. G. Harper, Proc. Phys. Soc. Sect. A 68, 874 (1955).

[25] D. R. Hofstadter, Phys. Rev. B 14, 2239 (1976).

[26] M. König, H. Buhmann, L. W. Molenkamp, T. Hughes, C.-X. Liu, X.-L. Qi, and S.-C. Zhang, J. Phys. Soc. Japan 77, 031007 (2008).

[27] Y. Hatsugai, Phys. Rev. B 48, 11851 (1993).

[28] Y. Hatsugai, Phys. Rev. Lett. 71, 3697 (1993).

[29] M. Fujita, K. Wakabayashi, K. Nakada, and K. Kusakabe, J. Phys. Soc. Japan 65, 1920 (1996).

[30] K. Nakada, M. Fujita, G. Dresselhaus, and M. S. Dresselhaus, Phys. Rev. B 54, 17954 (1996).

[31] B. Zhou, H.-Z. Lu, R.-L. Chu, S.-Q. Shen, and Q. Niu, Phys. Rev. Lett. 101, 246807 (2008).

[32] J. Linder, T. Yokoyama, and A. Sudbø, Phys. Rev. B 80, 205401 (2009).

[33] K. He, Y. Zhang, K. He, C.-Z. Chang, C.-L. Song, L.-L.
Wang, X. Chen, J.-F. Jia, Z. Fang, X. Dai, W.-Y. Shan, S.-Q. Shen, Q. Niu, X.-L. Qi, S.-C. Zhang, X.-C. Ma, and Q.-K. Xue, Nat. Phys. 6, 584 (2010).

[34] J.-H. Moon, S.-M. Seo, K.-J. Lee, K.-W. Kim, J. Ryu, H.-W. Lee, R. D. McMichael, and M. D. Stiles, Phys. Rev. B 88, 184404 (2013).

[35] J. Cho, N.-H. Kim, S. Lee, J.-S. Kim, R. Lavrijsen, A. Solignac, Y. Yin, D.-S. Han, N. J. J. van Hoof, H. J. M. Swagten, B. Koopmans, and C.-Y. You, Nat. Commun. 6, 7635 (2015).

[36] A. K. Chaurasiya, C. Banerjee, S. Pan, S. Sahoo, S. Choudhury, J. Sinha, and A. Barman, Sci. Rep. 6, 32592 (2016).

[37] A. Mook, J. Henk, and I. Mertig, Phys. Rev. B 91, 174409 (2015). 